strongest, with individual chapters covering stimulants, lysergic acid diethylamide and related drugs and the currently hot topic of cannabis. Psychosis and neurological conditions is also very comprehensively covered, as is psychosis and genetic disorders. The neurobiology of schizophrenia, functional imaging and neurological examination in schizophrenia are also discussed. Every chapter is well written and takes a modern evidence-based approach. Occasional tables and illustrations are nicely presented but these average only one or two per chapter. I can see only two weaknesses. First, the coverage of delirium (which the authors file under toxic psychosis) is rather brief. Second, there is almost nothing of note on dementia with Lewy bodies, even in the chapter on psychosis and neurodegenerative conditions. This is an omission that should be corrected for the next edition.

Overall, there is much to like in this volume and I highly recommend it to all psychiatrists who have tended to overlook this area in the past. Now there is no excuse.

Alex J. Mitchell Consultant in Psycho-oncology, Department of Liaison Psychiatry Brandon Unit, Leicester General Hospital, Gwendolen Road, Leicester LE5 4PW, UK. Email: alex.mitchell@leicspart.nhs.uk

doi: 10.1192/bjp.bp. 110.080390

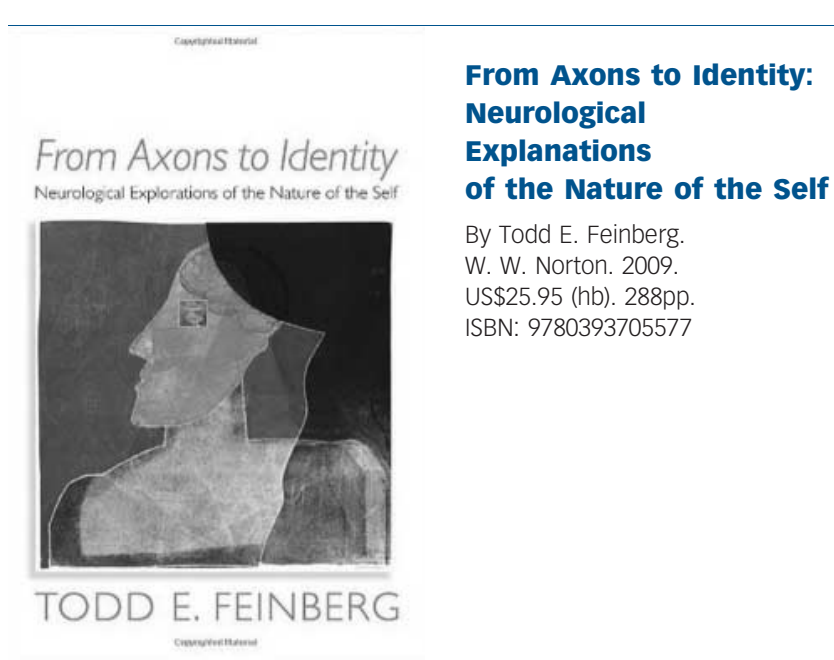

Todd Feinberg is an accomplished writer who manages to convey a lot of information in a relatively short compass. Laudably, he illustrates his points with transcripts of interviews with patients, particularly in the first half of the book.

From the clinical material he draws on, Feinberg has a great interest in what he terms 'neuropathologies of the self' anosognosia, asomatognosia and delusional misidentification, including Capgras and Fregoli syndromes. In the first half of the book, Feinberg's topic is one that those interested in psychosis and the philosophy of delusions are familiar with: how to give an account of delusions, and how, if at all, delusions are distinguished from other irrational beliefs and confabulations. Although the terminology is not employed, Feinberg works within the Maherian paradigm (a delusion being an explanation of an anomalous experience) and, like many contemporary researchers and theoreticians, adds in a second stage (traditionally, this second stage is a reasoning bias or neuropsychological deficit), which in combination with the odd experience leads to the delusion or unusual belief. For Feinberg, the main thesis is that damage to the brain (in the right frontal region particularly) engenders in people a reversion to utilising more primitive psychodynamic defence mechanisms, such as delusional projection. It seems the idea is that there is a genuine paralysis, for example, resultant on brain damage and this too can give rise to denial of disability (anosognosia) or projection (thinking the limb an imaginary friend or persecutor) via the reactivation of these primitive mechanisms.

The second half of the book seems largely independent of the first and is more ambitious. The author moves away from clinical concerns to notions of self, identity and consciousness and proposes the 'neural hierarchy theory of consciousness'. Here, Feinberg introduces the notion of 'nested hierarchy'.

The book as a whole is easy to read and of interest. However, as a stand-alone text, I felt it was not fully convincing in terms of the arguments offered and the data used. Feinberg has very compelling ideas which, if correct, are very important and I look forward to reading their exposition in future publications.

Matthew Broome Warwick Medical School, University of Warwick, Gibbet Hill, Coventry CV4 7AL, UK. Email: m.r.broome@warwick.ac.uk

doi: 10.1192/bjp.bp.109.075515

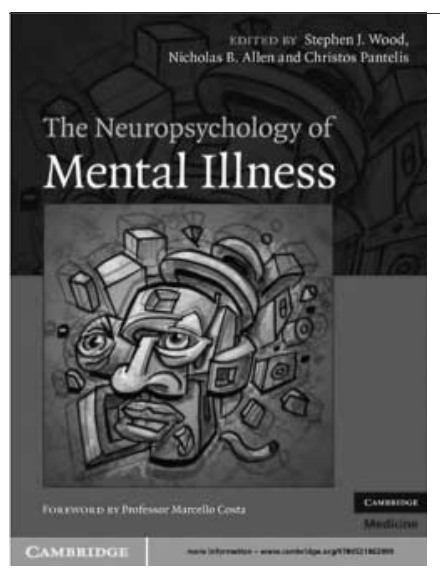

\section{The Neuropsychology of Mental Illness}

Edited by Stephen J. Wood, Nicholas B. Allen \& Christos Pantelis Cambridge University Press. 2009 £55 (hb). 443pp. ISBN: 9780521862899

One of the lesser noted revisions in the draft DSM-5 is a change in the definition of a mental disorder from 'a manifestation of a behavioural, psychological, or biological dysfunction' to one 'that reflects an underlying psychobiological dysfunction', highlighting the fact that we have increasingly come to regard neuropsychology as the best compromise in the vexatious mind-body problem and sometimes the reluctant redeemer of a medical specialty best known for its irreconcilable differences between objective and subjective world views.

Wood, Allen and Pantelis's comprehensive book aims to map out what this compromise has told us about the causes and effects of psychiatric disorder so far and, furthermore, what we have learnt about how to integrate neuropsychological approaches into the art and science of psychiatry. One component of this manifests itself as a series of competent and up-to-date review chapters on what we know about the neuropsychology of schizophrenia, as well as developmental, personality, eating, substance use, obsessive-compulsive and mood disorders, although I was left puzzled by the lack of chapters on anxiety disorders or posttraumatic stress disorder, both of which have received a great deal of neuropsychological attention.

The book also boasts a section of chapters dedicated to examining psychopathology from the perspective of normal 